\title{
Evaluation of Video Quality in Wireless Multimedia Sensor Networks
}

\author{
Mustafa Shakir, Obaid Ur Rehman, Zeeshan Abbas, Abdullah Masood, Wajeeha Shahid \\ Department of Electrical Engineering, COMSATS Institute of Information Technology, Islamabad, Pakistan
}

\begin{tabular}{l} 
Article Info \\
\hline Article history: \\
Received Feb 18, 2015 \\
Revised Nov 9, 2015 \\
Accepted Nov 22, 2015 \\
\hline Keyword: \\
Mean opnion score \\
Multimedia sensor network \\
Peak signal to noise ratio \\
Quality of experience
\end{tabular}

\begin{abstract}
Simulating wireless sensor networks, their implementation and evaluation, requires the use of a discrete event simulator. Omnet++ is quite a powerful simulator which supports concise and easy modeling of wired as well as wireless sensors environment. Scenarios involving multimedia transmissions with characteristics of video quality control and evaluation must be computed on the basis of Quality of Experience (QoE), which relies on user's perception to maintain the video quality. For the multimedia growth and awareness of future Wireless Multimedia Sensor Networks (WMSNs), it is quite necessary that the performance should be tested for different types of radio models. So varying the radio parameters may allow for the optimization and improvement of the video quality. In this paper, we have worked on Omnet++ framework for the evaluation and optimization of the performance of WMSN by using different radio models. The performance is evaluated based on the QoE metrics; i.e. Peak Signal-to-Noise ratio (PSNR) and Mean Opinion Score (MoS), which depend on user's perception to maintain the video quality.
\end{abstract}

Copyright (C) 2016 Institute of Advanced Engineering and Science. All rights reserved.

\section{Corresponding Author:}

Obaid Ur Rehman,

Department of Electrical Engineering,

COMSATS Institute of Information Technology,

Park Road, Chak Shehzad, Islamabad, Pakistan.

Email: obaid_iiec@yahoo.com

\section{INTRODUCTION}

Over the past few years wireless sensor networks have become an extremely important area in the research community. A sensing network of nodes constitutes to a powerful meld of sensing, processing as well as communicating technology. The versatility that wireless sensor networks offer has given rise to a multiple number of applications. These applications include monitoring and control of environments specifically industrial processes as well as health care, warfare, surveillance, traffic monitoring and enforcement, gaming as well as agriculture [1, 2]. Trends have been shifted from customary Wireless Sensor Networks which were capable of capturing scalar data only to WMSNs. These networks have great relevance to Internet of Things (IOT) which involves audio and video information such as multimedia, traffic and environmental monitoring to follow the events and changes in the environmental being monitored. These networks are outfitted with devices with some sensing capability that retrieves data from the environment for e.g. camera.

The multimedia content retrieved in such systems allows the users to perceive visually the impact of the scene being captured and being aware of the environment. So to comprehend the performance of the system based on user's perception, QoE metrics are to be used [3, 4]. By using the QoE metrics the performance, involving multimedia management and transmission, must be evaluated by keeping in view the users perception. These metrics include objective as well as subjective quality measures which in our case are 
PSNR and MoS respectively. The QoS is not able to interpret user's perception as efficiently as QoE since QoE can manage video flows with different properties and inter frame dependeies.

The key issues in choosing and developing proper routing protocols in WMSNs also include energy limitations, maintaining required QoS level, bandwidth oriented demand for particular applications alongwith low delay for selection between multiple available paths [5]. For the multimedia growth and awareness of future WMSNs, it is quite necessary that the performance should be tested from different types of radio models. So varying the radio parameters may allow for the optimization and improvement of the video quality. For the evaluation of different parameters firstly an event driven simulation is necessary before the actual deployment to help optimize time, cost as well as human resources.

One of the most important challenges faced by researchers is the development of efficient and flexible system software to make functional abstractions and information gathering from multimedia sensors. Simulating WMSNs requires the use of a discrete event simulator. Distributed image compression and proper transmission scheme in introduced in [6], which leverages to overcome scarce resources problem in sensor nodes and uneven energy consumption. The simulator that we used in our project is Omnet++. The basic novelity is frame loss, PSNR and MOS for different frames to evaluate video quality measurements and performance evaluation in a transmission. OMNET++ is quite a powerful simulator which supports concise and easy modeling of wired as well as wireless sensors environment. However, simple Omnet++ does not support video transmission so we used a separate framework known as M3WSN. This framework not only supports video transmission but also the evaluation and control of video content.

\section{OMNET++ FRAMEWORK FOR MULTIMEDIA TRANSMISSION}

There are number of frameworks that can be used with omnetpp for the creation of the required network. Different researchers worked on different frameworks for the purpose of multimedia streaming and the interesting thing is that every next framework uses the previous one. The frameworks that have the capability to transmit and receive the multimedia content within the network are Castalia, Wise-MNet, WVSN and M3WSN.

Castalia is a framework that is made to model algorithms for usual or traditional WSNs under practical and realistic communication conditions [7, 8]. The overall network architecture of Castalia framework consists of a wireless channel, physical process, and nodes as shown in the Figure 1.

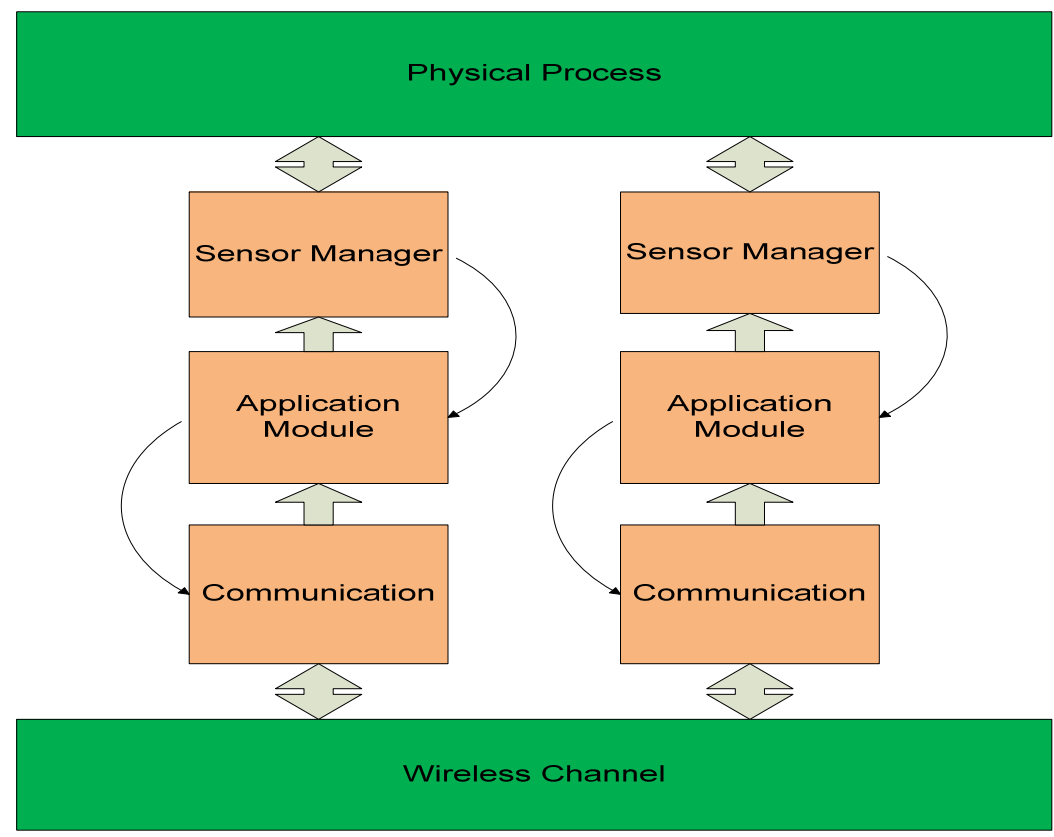

Figure 1. Castalia Network Architecture

The wireless channel connects different nodes with each other and also simulates the behavior of the wireless link. The physical process also connects the nodes within the network and feed the sensor manager 
of different nodes with data. Different modules compose a node, i.e., communication, application and sensor manager.

Wise-MNET provides an extension to some of Castalia's functions or features to generate simulation environment for WMSN [9]. WiSE-MNet provides a platform for the designing of the network prototcols.Wise-Mnet is designed for evaluating WMSN but doesn't provide QOE support and video control, by which we may evaluate the multimedia content from user's perspective. Moreover, it doesn't consider the realistic communication approach which should be taken into account that practically the wireless medium is unreliable and has to be considered for realistic communication approach. Also, WiSE-MNet does not support node mobility.

The WVSN model extends the older framework WiSE-MNet. In WVSN we measure the sensing range of the nodes using the field of view (FoV). In previous cases authers measure the sensing ranges assuming just like a disk that is an omnidirectional but in this the range is defined as a triangle with respect to the direction of the camera [10]. The sensing range depends on some of the factors like the direction of the camera (V), angle of view (alpha) and depth of view (d).

In this paper, we used Omnet++ framework for the evaluation and optimization of the performance using different radio models. The performance is comprehended based on the QoE metrics; i.e. PSNR and MoS which depend on user's perception to maintain the video quality.

M3WSN is new framework, which is based on Omnet++ and Castalia with the integrated properties of WiSE-MNet and WVSN along with its new functionalities for multimedia transmission and control [11]. This framework includes object detection, mobility, field of view FoV, cover-set and application criticality. The architecture of M3WSN framework is shown in Figure 2.

In WMSNs, we need to calculate/observe the behavior of motion of the object for its detection. So, for this purpose till now detection of objects is done by defining a range of the node. Any of the nodes occurs in its range can detect that node and the motion of the nodes may be of any kind i.e. linear, circular or random. In this model the scalar nodes can detect objects in its range using the omnidirectional way and the cameras are using the FoV concept for the realistic view. The multimedia transmission applications must have to calculate the video quality not just in terms of QoS but also in terms of QoE. Besides that if we provide a very good service of multimedia but if it is of high cost such that a normal person cannot afford then it has bad QoE.

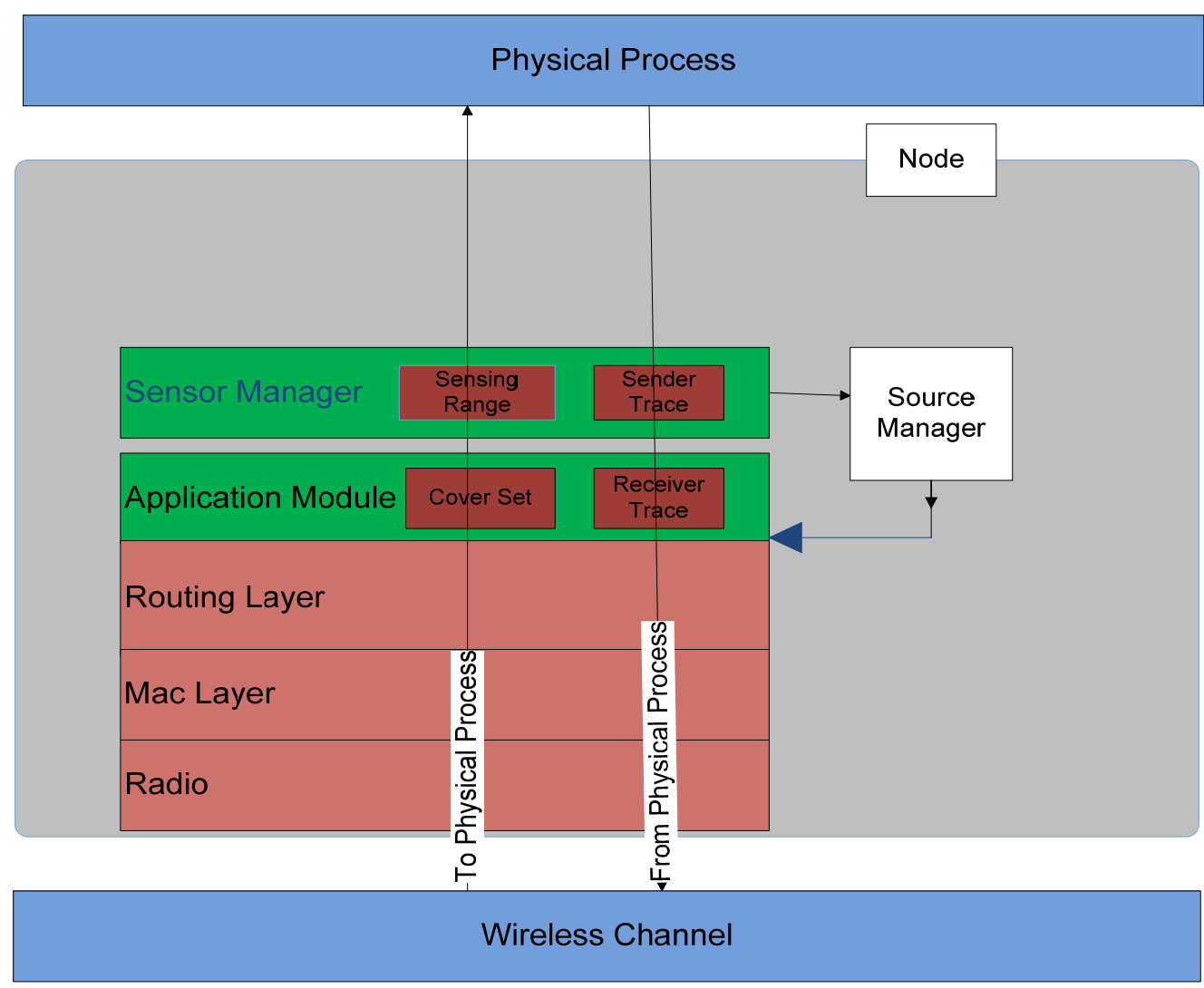

Figure. 2 M3WSN Node Architecture 


\section{MULTIMEDIA MANAGEMENT}

The size of multimedia packets is large enough such that its transmission is not easy as it requires high bandwidth and power. Multimedia transmission requires high data rates. Normally in videos the frame rate is 30 frames per second. In these frames different priorities are assigned to different frames [12]. The three different priority frames are, I-frames (Intra-Coded frames), P-frames (Predictive frames) and B-frames (Bi-predictive frames).

The loss of high priority frame can affect the result of video. On the decoder side the I-frames are used as the reference frame so the loss of I-frame may affect the whole video or the Group of Picture (GOP). Similarly, for the loss of Predictive frame it will affect the remaining frames in the GoP and the loss of Bipredictive frame can affect only the respective frame.

Castalia and the extensions (WiSE-MNet \& WVSN) not provided the control and evaluation of real video sequences. Therefore, M3WSN ported Evalvid which provides video related information, such as delay, jitter, frame type, received/lost and decoding errors etc.

EVALID as shown in Figure 3 is a part of M3WSN which is used to evaluate the video quality at the receivers end. To understand the working of EVALVID is essential so that scripts are written according to what to require and then the quality parameters could be observed. EVALVID basically work on both the ends of the simulation (i.e. sender and receiver end) so that the difference between the sent and received traces can be seen. Evalvid uses FFMPEG libraries which are a leading multimedia framework, able to encode, decode, transcode, de-multiplexer, multiplexer, stream, filter and play most stuff that humans and machines have created.

The video trace contains all the information about the frames that build the video and it is created only for single time. The information may include frame number, frame type, frame size and time to transmit each of the frames. Similarly, based on the information of the video trace file, for every video transmission the source node also has to create the sender trace file. The video trace file contains information about the packet size, packet id and the time stamp. These two trace files contain all the information for transmission at sender side and for further evaluation. On the other hand the sink node creates a receiver trace file for every received video. Like the sender trace file, the receiver trace file also contains information like packet size, packet id and time stamp.

As, the sensor manager module supports a camera in retrieving a video, therefore the creation of the sender traces are implemented on this module. On the other hand, due to the reason that application layer receives multimedia packets and reconstruct it, the receiver trace is created at this module.

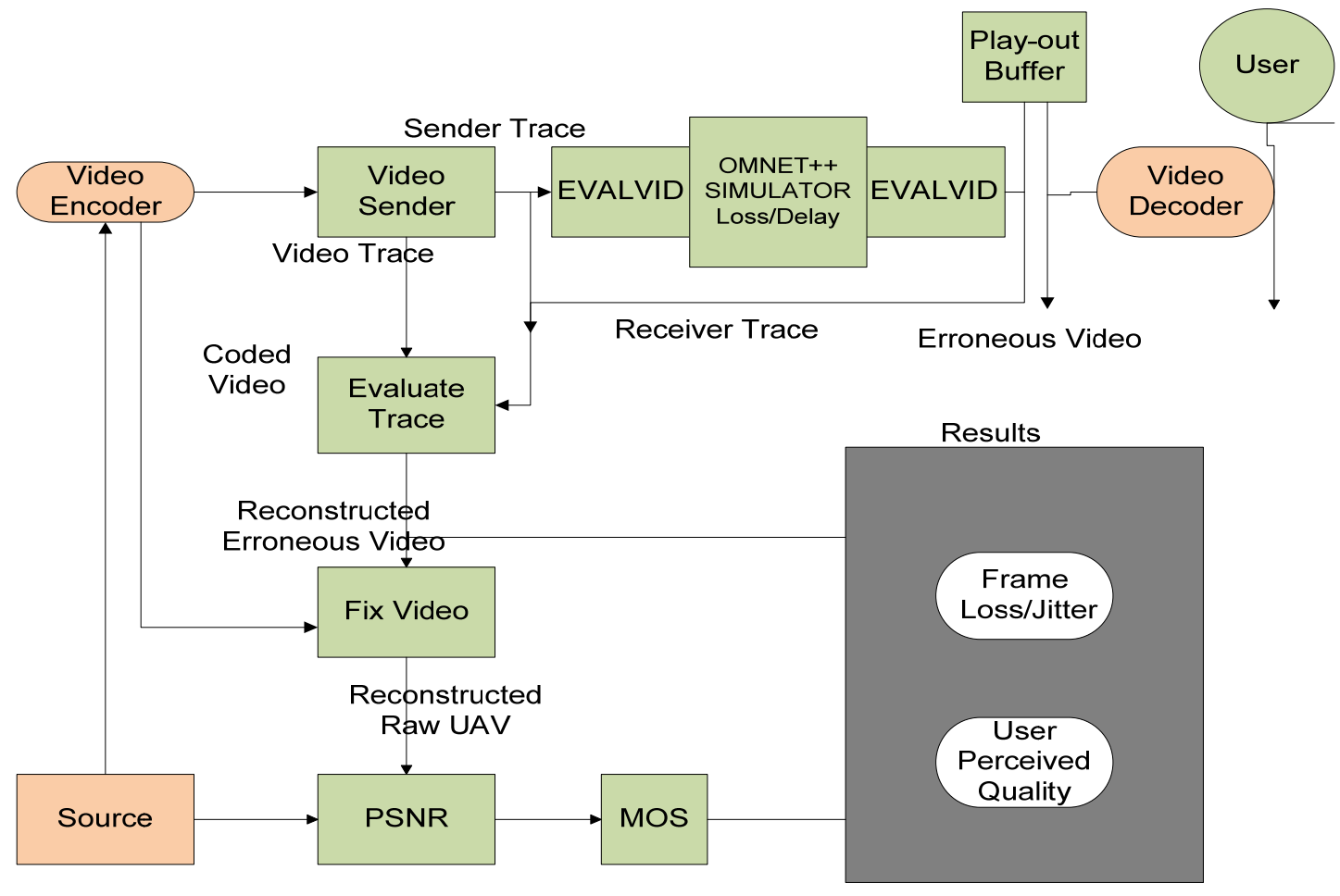

Figure 3. Evalvid Architecture 


\section{PERFORMANCE METRICS}

The two basic measures of quality that we are used in this paper are PSNR and on the basis of PSNR, we also measure MOS. PSNR is a term used for the ratio of maximum power of a signal to the maximum noise power which can degrade the quality of the signal and affect its fidelity. PSNR is mostly used to measure the quality of reconstructions during compressions [13]. In our case the input signal is the original video and the errors are introduced by the compression through codecs. PSNR is the human perception approximation about the reconstructed video. Generally higher PSNR means the quality of reconstruction is high and vice versa.

$$
P S N R=10 \log _{10}\left[\frac{M A X^{2}}{M S E}\right]
$$

Table 1 defines the range of PSNR values, shows the quality level.

Table 1. Relation of PSNR and video quality

\begin{tabular}{cc}
\hline PSNR & QUALITY \\
\hline$>37$ & Excellent \\
$31-37$ & Good \\
$25-31$ & Fair \\
$20-25$ & Poor \\
$<20$ & Bad \\
\hline
\end{tabular}

Video quality measurements must be based upon the human perception i.e. the users, by watching the video comments about it as good or bad. This type of video quality measurements is also called as subjective impression of the user and provides more information. However, it is very costly and time consuming that humans start to watch the videos from start to the end and share their comments about that, it requires high manpower. So, these types of subjective methods are defined by ITU in detail. Table 2 show the MOS which is one of the subjective quality metrics describing the human perception [14].

Table 2. Relations of MOS and video quality

\begin{tabular}{cc}
\hline MOS & QUALITY \\
\hline 5 & Excellent \\
4 & Good \\
3 & Fair \\
2 & Poor \\
1 & Bad \\
\hline
\end{tabular}

$$
M S E=\sum_{i=0}^{m-1} 1 \sum_{j=0}^{i-1}[I(i, j)-K(i, j)]^{2}
$$

MSE is the mean squared error, I is the original image, $\mathrm{K}$ is the compressed image and $\mathrm{MxN}$ is the dimension of both images.

\section{RADIO MODELS}

The CC2420 and CC1000 are two chips used for different purposes like very low power data transmitters and receivers, home automation, wireless alarm and security, game controllers and automatic meter reading etc. The parameters of both radios differ a lot which in turns affects the results as well. The parameters are shown in Table 3. 
Table 3. Parameters of radio models

\begin{tabular}{lcc}
\hline \multicolumn{1}{c}{ Parameters } & CC2420 & CC1000 \\
\hline Data rate (kbps) & 250 & 19.2 \\
Modulation & PSK & FSK \\
Bits/symbol & 4 & 1 \\
Bandwidth (MHz) & 20 & 10 \\
Noise Bandwidth & 194 & 30 \\
(MHz) & & \\
Noise floor (dBm) & -100 & -105 \\
Sensitivity (dBm) & -95 & -98 \\
Power consumed (mW) & 62 & 22.2 \\
\hline
\end{tabular}

Comparison of data rate and the bandwidth is evaluated through Shannon capacity formula:

$$
C=B \log _{2}(1+S N R)
$$

The bandwidth is directly proportional to the capacity. So, in CC2420 as the data rate is 250kbps therefor its bandwidth is also high i.e. $20 \mathrm{MHz}$ while in CC1000 the data rate is $19.2 \mathrm{kbps}$ which is low compared to the radio CC2420 therefor the required bandwidth is also low i.e. $10 \mathrm{MHz}$.

\section{SIMULATION RESULTS}

We analyze the frame loss percentage and QoE metrics like PSNR and MOS of both radios. Analysis of results reveils that the performance of radio CC1000 is almost double as compared to the radio CC2420.

The Figure 4 and Figure 5 show the frame loss percentage of radio model CC2420 and CC1000 with respect to different powers in $\mathrm{dBm}$. Figure 4 shows that in radio CC2420 the performance starts to degrade from $-4 \mathrm{dBm}$ however, Figure 5 portray the performance is degrading after $-8 \mathrm{dBm}$ for radio CC1000. In this sense, we are able to find the threshold power level for a reliable multimedia transmission for different radios. Videos consists of frames with different priorities like I, P and B and the loss of high priority frames distorts the video much more compared to the low priority frames. The Figure 4 and Figure 5 also show the individual frame losses of I, P and B and also their average. I frame loss percentage is high as compared to others because I frame is used as the reference frame for the others and has high priority. If any of the $\mathrm{P}$ or $\mathrm{B}$ frames are lost these frames can be reconstructed by using their reference frame therefore, their probability of loss is low. These frames have not too much effect on the quality However, on the other side if I frame is lost it cannot be reconstructed in any case.

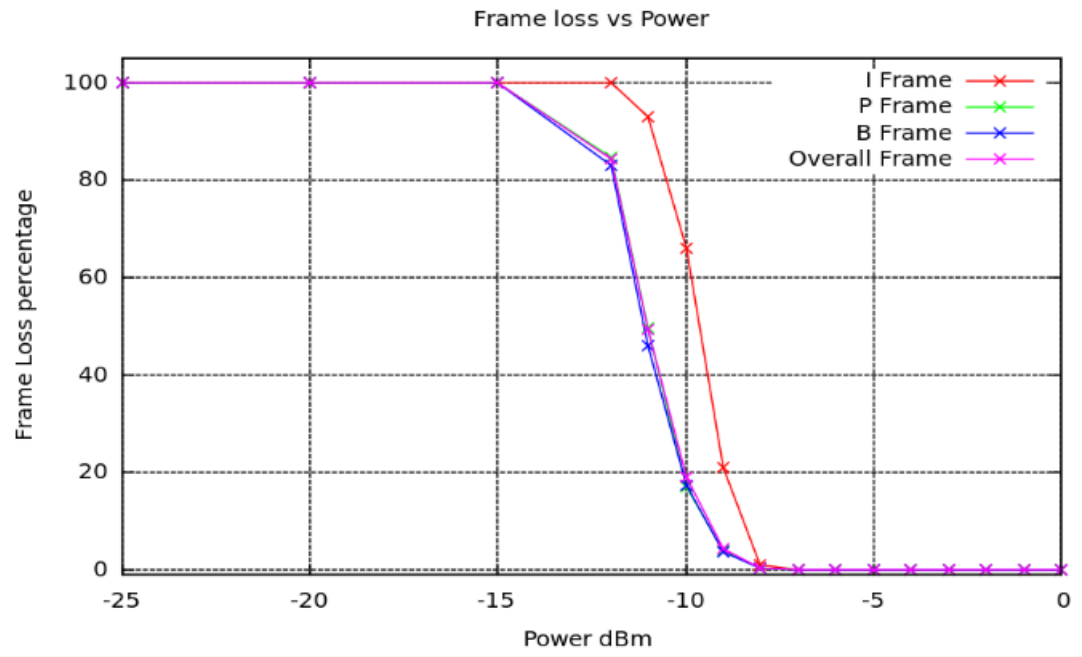

Figure 4. Frame loss for CC2420 


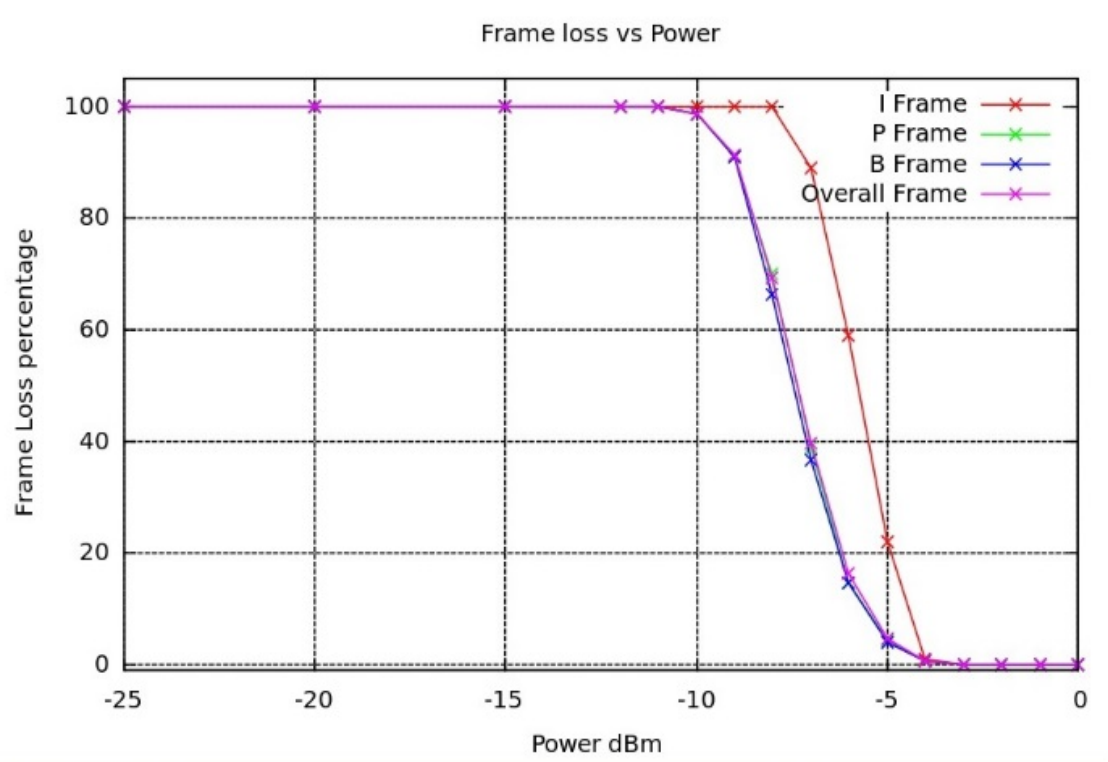

Figure 5. Frame loss for CC1000

PSNR is mostly used to measure the quality of reconstructions during compressions. As we mentioned earlier that the performance of CC1000 is double as compared to CC2420, it can be observed in Figure 6 and Figure 7 that the PSNR at $-3 \mathrm{~dB}$ in radio CC2420 is same as the PSNR at -6dB in radio CC1000. Also the performance at $-3 \mathrm{~dB}$ and $-6 \mathrm{~dB}$ in radio $\mathrm{CC} 1000$ is similar therefor their graphs are overlapping in Figure 6. Similarly the performance at $-9 \mathrm{~dB}$ and $-15 \mathrm{~dB}$ in radio CC2420 is similar and their graphs are overlapping in Figure 7.

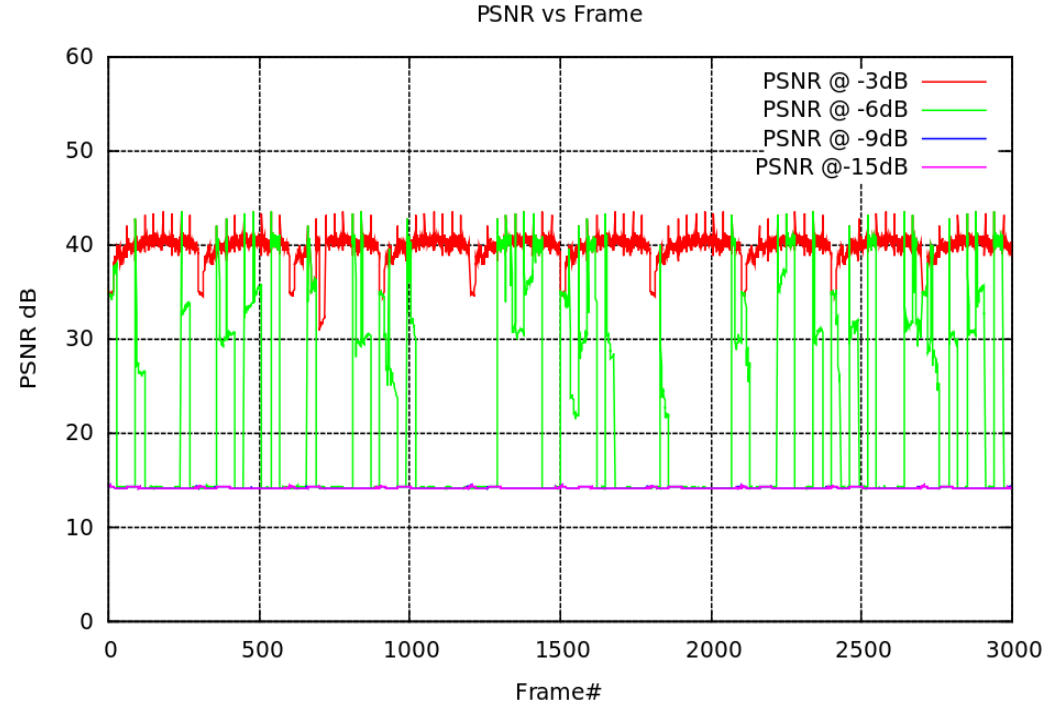

Figure 6. Peak Signal to Noise Ratio (CC2420) 


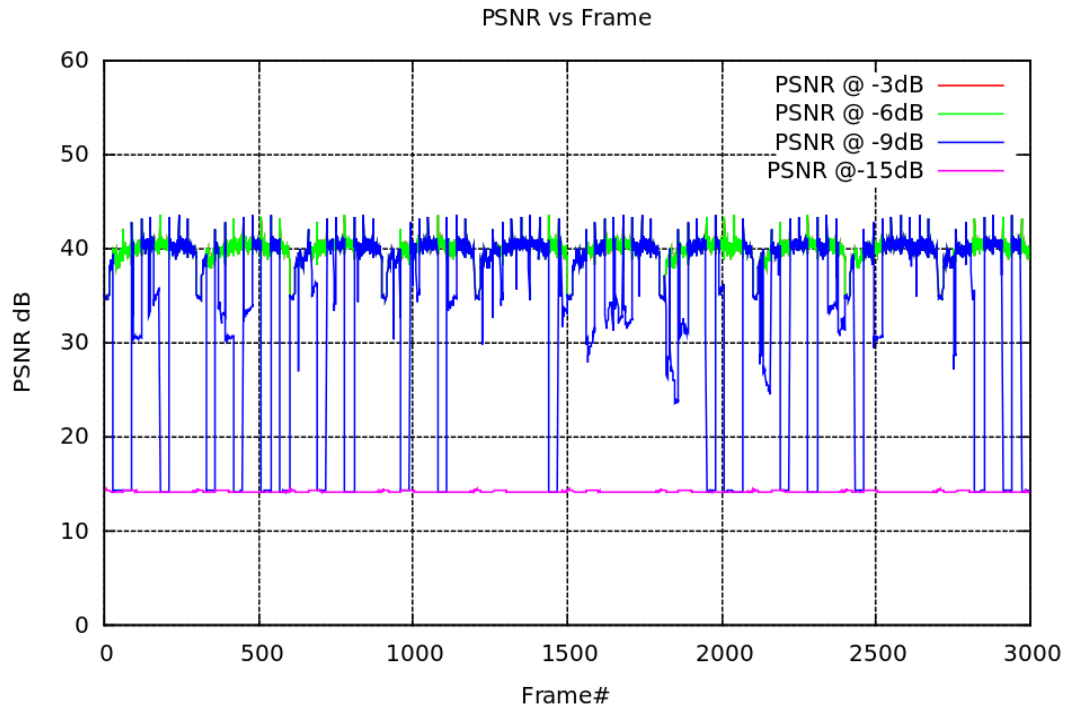

Figure 7. Peak Signal to Noise Ratio (CC1000)

MOS is one of the subjective quality metrics which describes the human perception about the video whether it is Excellent, Good, Fair, Poor, or Bad. The MOS graphs are directly reflecting the above mentioned graphs of frame loss and the PSNR. Frame loss in radio CC2420 starts from -4dB till this point there was no frame loss means excellent quality of the video. So this can be directly observed here in Figure 8. The MOS till -4dB that show the human perception of excellent video quality is almost 5 . On the other hand, Figure 9 shows the MOS is almost equal to 5 till $-8 \mathrm{~dB}$ reflecting the point that the frame loss starting from this point.

Lastly, Figures 10-13, portray the screenshots of the video at a fixed time (2 seconds) at different powers for both radios CC2420 and CC1000 showing the quality of the video received. Since the performance of CC1000 is better at $-6 \mathrm{~dB}$ and $-9 \mathrm{~dB}$ respectively than CC2420. The best result is at $-6 \mathrm{~dB}$ of radio CC1000 as at this point we can see the MOS is almost 5 and the PSNR is also greater than 37.

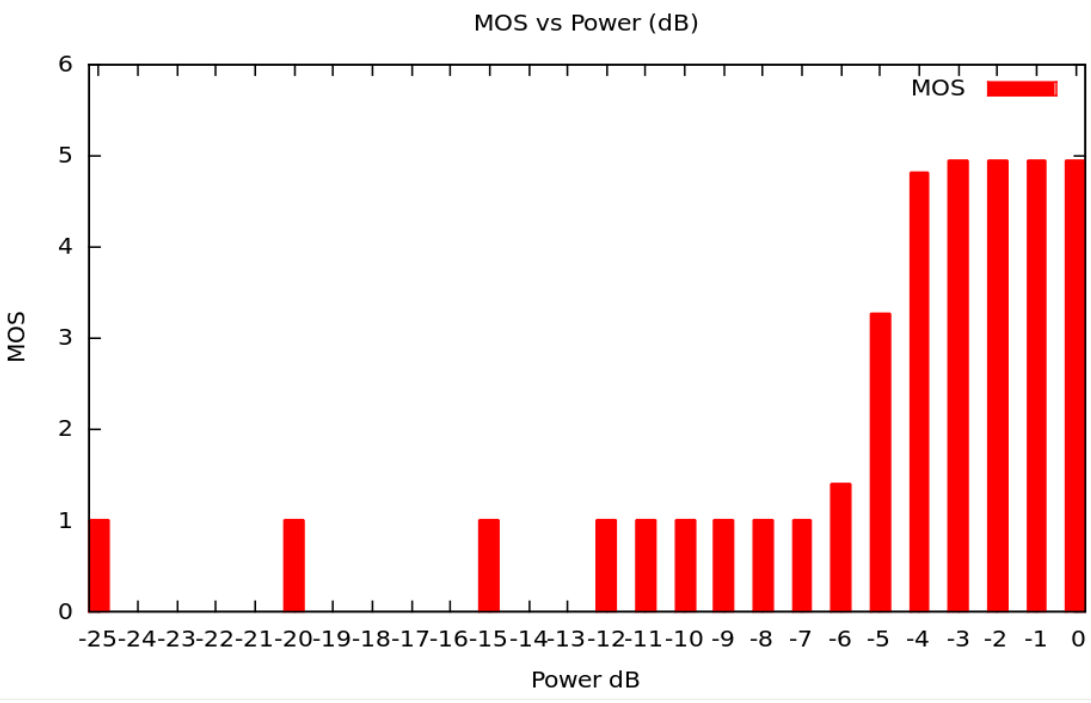

Figure 8. Mean Opnion Score (CC2420) 
MOS vs Power (dB)

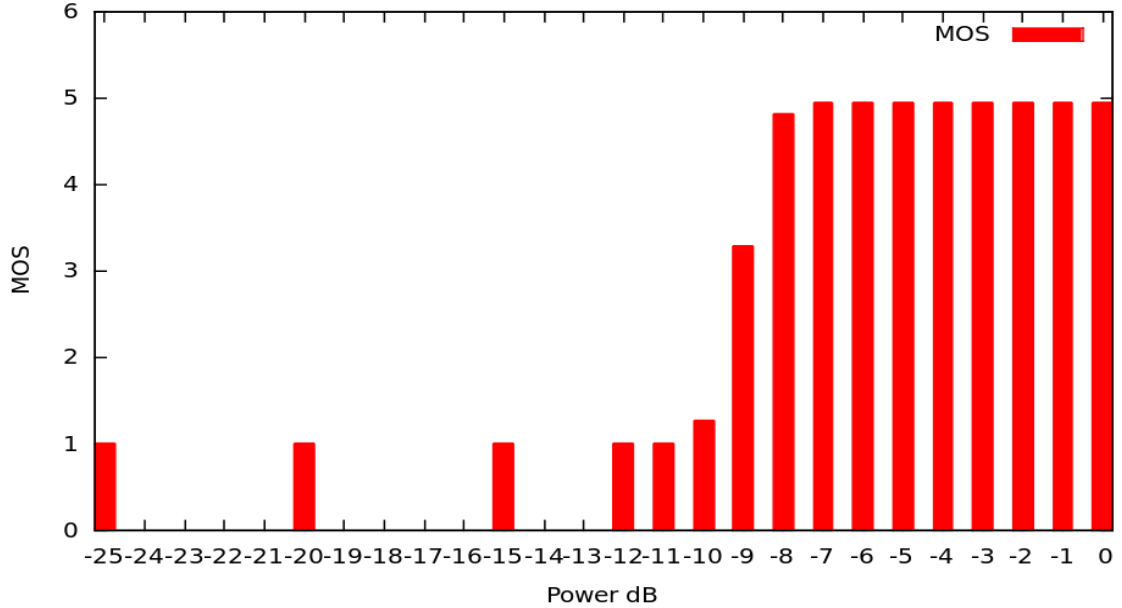

Figure 9. Mean Openion Score (CC1000)

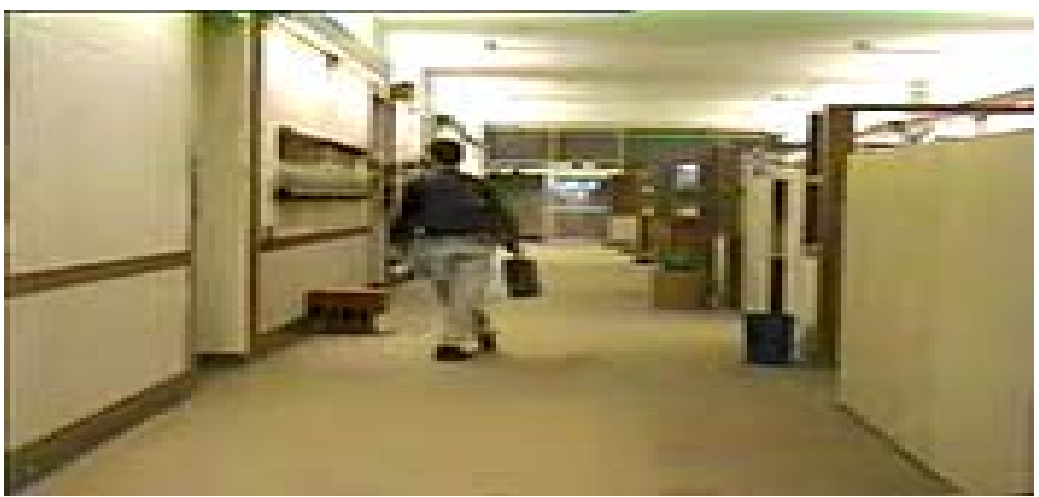

Figure 10. Video Snap at -6dB (CC2420

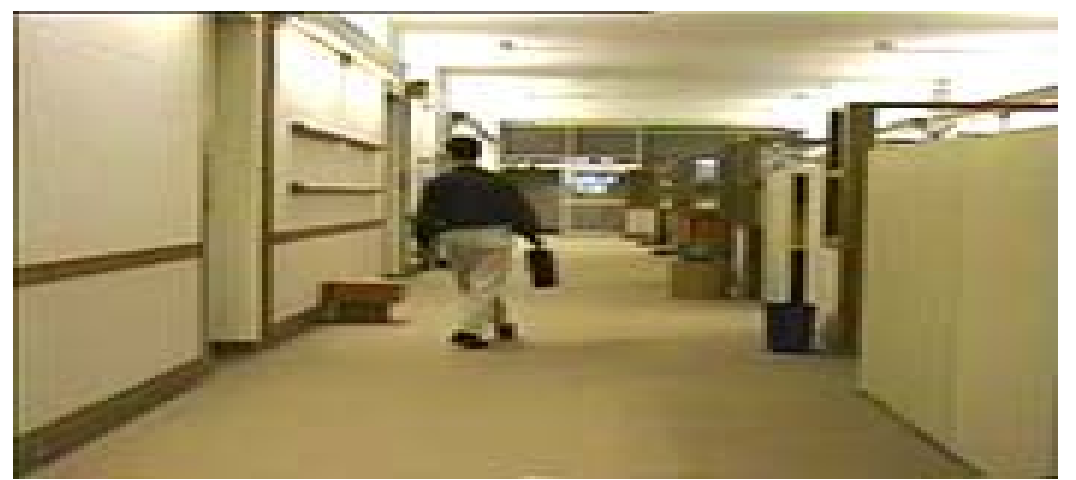

Figure 11. Video snap at -6dB (CC1000) 


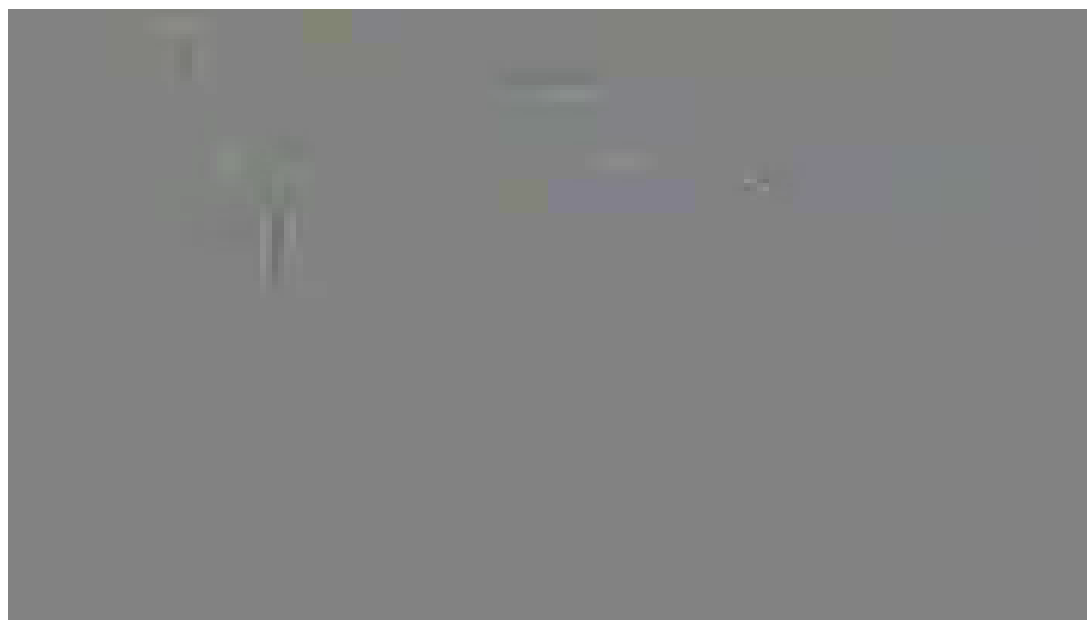

Figure 12. Video snap at -9dB (CC2420)

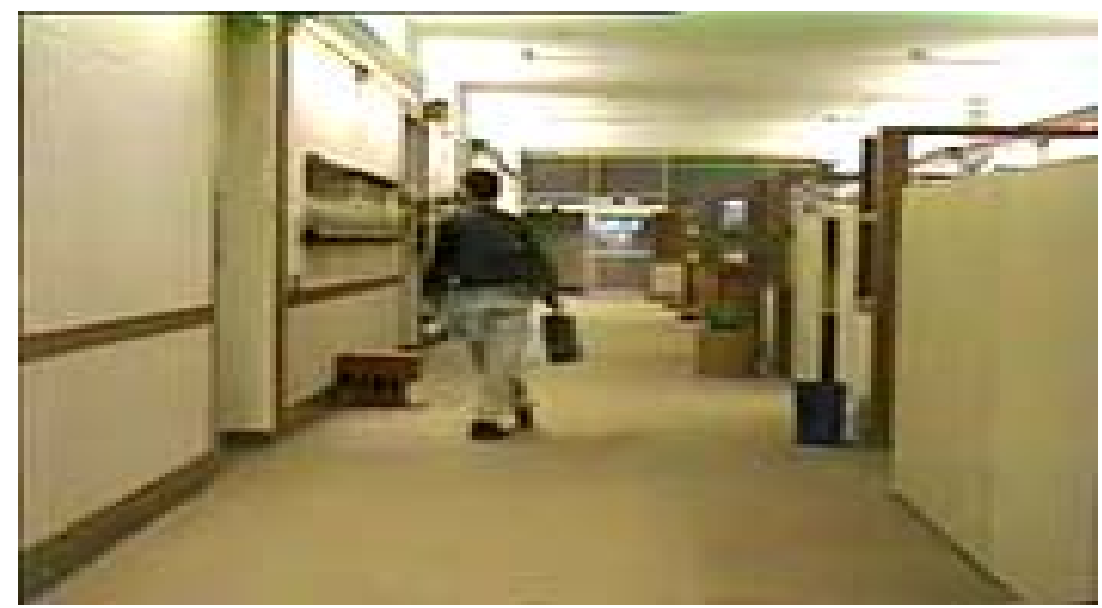

Figure 13. Video snap at -9dB (CC1000)

\section{CONCCLUSIONS}

In our work, we evaluated multimedia content from user's perspective and managed to maintain a fair QoE of video at the receiver end. Omnet++ framework analysis reveiled the video quality based on QoE metrics i.e PSNR and MoS. The results clearly prove that the radio model CC1000 provide a better video quality as compared to CC2420. The threshold power at which the frame loss percentage is sufficiently optimized is also at a less value in case of CC1000.

\section{REFERENCES}

[1] Sharif, Atif, Vidyasagar Potdar, and Elizabeth Chang, "Wireless multimedia sensor network technology: A survey", Industrial informatics, 2009. INDIN 2009. 7th IEEE international conference on. IEEE, 2009.

[2] Akyildiz, Ian F., Tommaso Melodia, and Kaushik R. Chowdhury, "Wireless multimedia sensor networks: Applications and testbeds", Proceedings of the IEEE, 96.10 (2008): 1588-1605.

[3] Cerqueira, Eduardo, et al, "Recent advances in multimedia networking", Multimedia Tools and Applications, 54.3 (2011): 635-647.

[4] Mehmood, Rashid, Raad Alturki, and Sherali Zeadally, "Multimedia applications over metropolitan area networks (MANs)", Journal of Network and Computer Applications, 34.5 (2011): 1518-1529.

[5] Sun, Enyan, Chuanyun Wang, and Feng Tian, "A Survey on Multi-path Routing Protocols in Wireless Multimedia Sensor Networks", TELKOMNIKA Indonesian Journal of Electrical Engineering, 12.9 (2014): 6978-6983.

[6] Sun, Enyan, et al, "Distributed Image Compression and Transmission Scheme in Wireless Multimedia Sensor Networks", TELKOMNIKA Indonesian Journal of Electrical Engineering, 12.1 (2014): 661-668. 
[7] Boulis, Athanassios, "Castalia User Manual", Online: http://castalia.npc.nicta.com.au/pdfs/CastaliaUserManual.pdf, (2009).

[8] Rastegarnia, Adib, and Vahid Solouk, "Performance evaluation of castalia wireless sensor network simulator", Telecommunications and Signal Processing (TSP), 2011 34th International Conference on. IEEE, 2011.

[9] Nastasi, Christian, and Andrea Cavallaro, "WiSE-MNet: an experimental environment for wireless multimedia sensor networks", (2011): 34-34.

[10] Sabokrou, Mohammd, Mahmood Fathy, and M. Hoseni, "Intelligent Target Tracking in Wireless Visual Sensor Networks", Computer and Knowledge Engineering (ICCKE), 2012 2nd International eConference on. IEEE, 2012.

[11]] Rosário, Denis, et al, "An omnet++ framework to evaluate video transmission in mobile wireless multimedia sensor networks", Proceedings of the 6th International ICST Conference on Simulation Tools and Techniques. ICST (Institute for Computer Sciences, Social-Informatics and Telecommunications Engineering), 2013.

[12] Karimi, Elham, and Behzad Akbari, "Priority Scheduling for Multipath Video Transmission in WMSNS", International Journal of Computer Networks \& Communications (IJCNC), 5.6 (2013).

[13] Huynh-Thu, Quan, and Mohammed Ghanbari, "Scope of validity of PSNR in image/video quality assessment", Electronics letters, 44.13 (2008): 800-801.

[14] Huynh-Thu, Quan, and Mohammed Ghanbari, "Scope of validity of PSNR in image/video quality assessment", Electronics letters, 44.13 (2008): 800-801.

\section{BIOGRAPHIES OF AUTHORS}

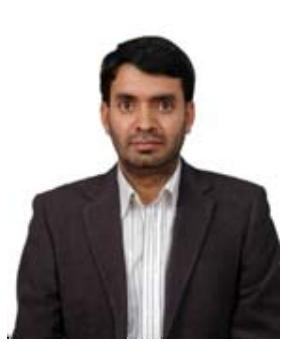

Dr. Mustafa Shakir got his $\mathrm{PhD}$ in Information and Communication Engineering from BeijingUniversity of Posts and Telecommunications, China in 2008. He is an Assistant Professor at Department of Electrical Engineering, COMSATS Institute of Information Technology, Islamabad since 2010. He has also worked at Pakistan Telecommunications Company Limitedand conducted sessions and trainings on Broadband Access, Next Generation Networks, IPTV, Data Communications and Understanding Telecom Networks and Services. His areas of interestinclude wireless networks and communication systems, ad hoc networks, sensor networks and next generation networks.

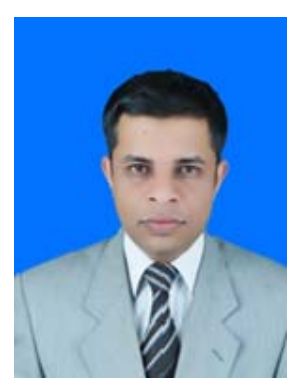

Mr. Obaid Ur Rehman received the M.S degree in Electrical Engineering in 2013 from the Facultyof Electrical Engineering at the COMSATS, Institute of Information Technology, Islamabad, Pakistan. He received the B.S degrees in Electrical Engineering in 2010 from the Riphah International University Islamabad, Pakistan. Currently, he is a lecturer at the COMSATS, Institute of Information Technology. His current research interests include wirelesss communication and networking, multimedia communication, ad hoc and sensor networks.

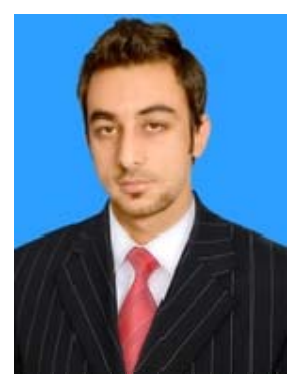

Mr. Zeeshan Abbas has done BS in Electrical (Telecommunication) Engineering from COMSATS Institute of Information Technology, Islamabad. His research interests include Network Security, Wireless Routing protocols. 


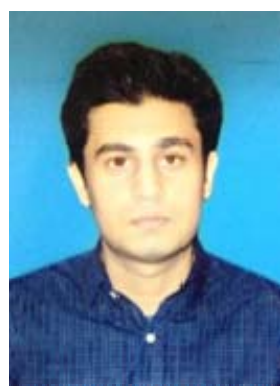

Abdullah Masood has done Electrical Engineering from COMSATS Istitute of Information and Technology specialized in Telecommunication. He is currently working for Nayatel Pvt. LTD as 'Network Support engineering' which is the first south Asian Fiber-to-the-home Private Company. His research interest includes Wireless Sensor Networks and Wireless Multimedia Sensor Networks.

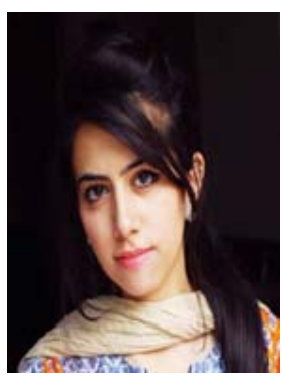

Engr. Wajeeha Shahid has done BS Eletrical (Telecom) Engineering from COMSATS. Her research interests include wireless networks, evaluation and optimization of video quality in Multimedia Wireless Sensor Networks. 